\title{
Electrically conductive bacterial cellulose composite membranes produced by the incorporation of graphite nanoplatelets in pristine bacterial cellulose membranes
}

\author{
T. Zhou ${ }^{*}$, D. Chen ${ }^{1}$, J. Jiu', T. T. Nge ${ }^{2}$, T. Sugahara ${ }^{2}$, S. Nagao ${ }^{2}$, H. Koga ${ }^{2}$, M. Nogi ${ }^{2}$,

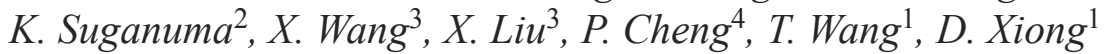

\begin{abstract}
${ }^{1}$ School of Materials Science and Engineering, Nanjing University of Science and Technology, 210094 Nanjing, China ${ }^{2}$ Institute of Scientific and Industrial Research, Osaka University, Mihogaoka 8-1, Ibaraki, 567-0047 Osaka, Japan ${ }^{3}$ Key Laboratory for Soft Chemistry and Functional Materials of Ministry Education, Nanjing University of Science and Technology, 210094 Nanjing, China

${ }^{4}$ National Key Laboratory of Science and Technology on Micro/Nano Fabrication, Key Laboratory for Thin Film and Microfabrication of the Ministry of Education, Research Institute of Micro/Nano Science and Technology, Shanghai Jiao Tong University, 200240 Shanghai, China
\end{abstract}

\begin{abstract}
Graphite nanoplatelets (GNPs) were utilized to improve the electrical conductivity of pristine bacterial cellulose (BC) membranes. By physical and chemical methods, flake-shaped GNPs, weaving through the surface layer of web-like cellulose nanofibrils, were indeed fixed or trapped by the adjacent nanofibrils in the BC surface network, for comparison, rod-shaped multi-walled carbon nanotubes (MWCNTs) were homogeneously inserted into BC membrane through the pore structures and tunnels within the $\mathrm{BC}$ membrane. Strong physical and chemical interaction exists between the BC nanofibrils and the particles of GNP or MWCNT even after $15 \mathrm{~h}$ sonication. BC membrane with $8.7 \mathrm{wt} \%$ incorporated GNPs reached the maximum electrical conductivity of $4.5 \mathrm{~S} / \mathrm{cm}$, while $13.9 \mathrm{wt} \% \mathrm{MWCNT} / \mathrm{BC}$ composite membrane achieved the maximum electrical conductivity of $1.2 \mathrm{~S} / \mathrm{cm}$. Compared with one dimensional (1-D) MWCNTs, as long as GNPs inserted into BC membranes, the 2-D reinforcement of GNPs was proven to be more effective in improving the electrical conductivity of BC membranes thus not only break the bottleneck of further improvement of the electrical conductivity of BC-based composite membranes but also broaden the applications of BC and GNPs.
\end{abstract}

Keywords: polymer composites, nanocomposites, electrical conductivity, graphite nanoplatelets, bacterial cellulose membrane

\section{Introduction}

Bacterial cellulose (BC), bacteria-produced natural biopolymer composed of ribbon-shaped nanofibers ( $<100 \mathrm{~nm}$ wide), is distinguished by its extensive hydrogen bonding-stabilized ultrafine porous threedimensional (3-D) weblike structure $[1,2]$. Since each $\mathrm{BC}$ nanofiber is a bundle of cellulose nanofibrils which are aggregates of semicrystalline extended cellulose chains, the coefficient of thermal expan- sion of $\mathrm{BC}$ nanofibers in the axial direction is similar to that of glass (as small as $0.1 \mathrm{ppm} / \mathrm{K}$ ), the Young's modulus $(\sim 138 \mathrm{GPa})$ and tensile strength (estimated to be at least $2 \mathrm{GPa}$ ) of BC nanofibers are almost equal to those of aramid fibers (e.g., Kevlar, a well-known high-strength fiber) [3, 4]. As a renewable and natural resources-based biodegradable polymers with great industrial potential (low cost production on a large scale), especially on account of

\footnotetext{
*Corresponding author, e-mail: ztltianle999@hotmail.com (C) BME-PT
} 
its unique structure and notable properties that can be converted into high value products, $\mathrm{BC}$, nowadays, has attracted rapidly growing worldwide scientific interest in which particular attention has been paid to $\mathrm{BC}$-based advanced functional materials, such as electrically conductive BC-based composite membranes [1,2].

Carbon nanotubes (CNTs), a type of one-dimensional (1-D) carbon nanomaterials with remarkable electrical conductivity, have been utilized in improving the electrical conductivity of insulating $\mathrm{BC}$ membranes for use in electronics such as electrodes [5-10]. Yoon and coworkers [5, 6] dipped BC membrane in a surfactant-stabilized $0.05 \mathrm{wt} \%$ multiwalled CNT (MWCNT) well-dispersed aqueous solution for $24 \mathrm{~h}$, consequently MWCNTs were found to be inserted in the $\mathrm{BC}$ membrane and the electrical conductivity of MWCNT/BC composite membranes with different MWCNTs' surfactants, cationic cetyltrimethylammonium bromide (CTAB) or sodium dodecylbenzenesulfonate (NaDDBS), greatly increased to $1.4 \times 10^{-1}$ and $4.2 \times 10^{-1} \mathrm{~S} / \mathrm{cm}$, respectively. Jung et al. [7] also utilized $\mathrm{BC}$ membrane as the template to deposit MWCNTs uniformly, then used silk fibroin as coating material for the MWCNT-incorporated BC membrane, the electrical conductivities of the resulting composite membranes consequently achieved respectively $5.3 \times 10^{-3}$ and $3.6 \times 10^{-3} \mathrm{~S} / \mathrm{cm}$ from CTAB-stabilized 0.03 or $0.05 \mathrm{wt} \%$ MWCNT aqueous dispersion (immersion time was unaccounted). Kim et al. [8] fabricated electrically conductive composite membrane with a surface resistance of $2.8 \mathrm{k} \Omega / \mathrm{sq}$ by immersing BC membrane in a NaDDBS-stabilized $0.01 \mathrm{wt} \%$ single-walled CNTs (SWCNTs) aqueous dispersion for $3 \mathrm{~h}$. They also fabricated composite membranes containing similar amount of purified MWCNTs or Ag-doped MWCNTs by immersing BC membranes in CTAB-stabilized $0.03 \mathrm{wt} \%$ MWCNTs or Ag-doped MWCNTs aqueous dispersions for $24 \mathrm{~h}$, which resulted in comparable electrical conductivities, $2.5 \times 10^{-3}$ and $3.1 \times 10^{-3} \mathrm{~S} / \mathrm{cm}$, respectively [9]. Yun et al. [10] introduced Pt-incorporated MWCNTs into MWCNT/BC composite membrane to improve the performances of electrodes of proton exchange membrane fuel cells and the final electrode had a sheet resistance of $80 \Omega / \mathrm{sq}$.

Furthermore, graphenes, a type of two-dimensional (2-D) carbon nanomaterials with remarkable electrical conductivity, were also used to improve the electrical conductivity of insulated $\mathrm{BC}$ membranes for use in biosensor and tissue engineering. Feng et al. [11] dissolved $\mathrm{BC}$ in $\mathrm{NaOH}$ solution, mixed graphene oxide (GO) and $\mathrm{BC}$ aqueous suspension together and finally obtained $\mathrm{GO} / \mathrm{BC}$ composite membrane with a electrical conductivity of $1.1 \times 10^{-4} \mathrm{~S} / \mathrm{cm}$ by filtering the colloidal aqueous suspension of $\mathrm{GO}$ and $\mathrm{BC}$.

CNT has great challenge in composite applications especially due to its expensive production cost, particularly for the purifying process; meanwhile graphene, a single layer of hexagonally arrayed $\mathrm{sp}^{2}$ bonded carbon, also has great challenge in composite applications since graphenes tend to form irreversible agglomerates or even restack to form graphite through van der Waals interactions during the production of bulk-quantity monolayer graphenes and their dispersion into matrices [12]. Recently, graphite nanoplatelet (GNP), a 2-D layered graphitic nanofiller composed of stacked graphene sheets, is considered as an alternative carbon-based electrically conductive nanofiller because of its comparable electrical conductivity, lower cost than CNT due to the abundance of naturally existing graphite as the source material for GNP, and easier large-scale processes than monolayer graphene since only one dimension, i.e. thickness, of the 2-D GNP falls within the nanoscaled range. However, using GNPs to improve the electrical conductivity of insulating $\mathrm{BC}$ membranes has not been considered yet. In this study, a new kind of BC-based composite, i.e. GNP/ $\mathrm{BC}$ composite membranes with $\mathrm{BC}$ acting as a supporting material to deposit the well-dispersed GNPs, was prepared, not only to break the bottleneck of further improving the electrical conductivity of BCbased composite membranes, but also broaden the applications of BC and GNPs.

\section{Experimental}

\subsection{Materials}

BC wet membranes $\left(20 \times 20 \mathrm{~mm}^{2}, 3 \mathrm{~mm}\right.$ thick $)$, the bacterial-cell debris of which had already been removed by purified in sodium hydroxide after produced by Gluconacetobacter xylinum, were supplied by Hainan Yida Food Industry Co., Ltd., Hainan, China. The as-received gel-like white purified BC membranes were firstly neutralized by thoroughly washing with absolute ethanol before being stored in absolute ethanol in a sealed container at $4^{\circ} \mathrm{C}$. As shown in Figure 1, BC utilized in this work has an 


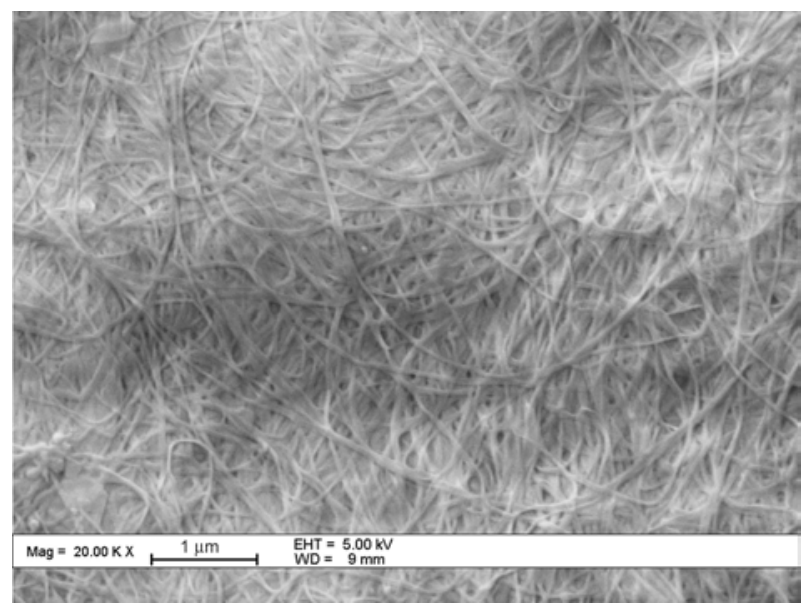

Figure 1. FESEM micrograph of the surface of air-dried BC membrane

ultrafine network structure consisted of a random assembly of nanofibrils with an average width of $\sim 50 \mathrm{~nm}$.

Graphite intercalation compound (GIC) particles (sized at $500 \mu \mathrm{m}$ ), composed of natural graphite particles intercalated by sulfuric acid, were supplied by Xinfangyuan Co. Ltd., Zaozhuang, China. According to the supplier's specification, the content of intercalants was about $15 \mathrm{wt} \%$ and the carbon content was higher than $99 \%$.

MWCNTs, rather than SWCNTs or double-walled CNTs, have been predominantly used due to their relatively lower cost, better availability and easier dispersability. Purified MWCNTs utilized in this work were provided by Chengdu Organic Chemicals Co., Ltd., Chengdu, Chinese Academy of Sciences. According to the supplier's specification, the purified MWCNTs with an aspect ratio of 125-400 (50-80 $\mathrm{nm}$ in diameter and 10-20 $\mu \mathrm{m}$ in length) were synthesized by catalytic chemical vapor deposition. The purity and the specific surface area of the MWCNTs are greater than $95 \%$ and $40 \mathrm{~m}^{2} / \mathrm{g}$, respectively. Micrographs of the rod-shaped MWCNTs can be seen in our previous works [13]. During purification, MWCNTs were refluxed with concentrated nitric acid to remove catalyst particles, which may also increase the amount of oxygen chemisorbed on the surface of MWCNTs and, therefore, cause more oxygenous functional groups formed on the surface of MWCNTs. The Fourier-transform infrared (FTIR) spectrum, as shown in Figure 2, showed that there is a mass of hydroxyl groups $(-\mathrm{OH})$ on the surface of as-received purified MWCNTs. Before stored in desiccators, MWCNTs were dried at $110^{\circ} \mathrm{C}$ for $24 \mathrm{~h}$

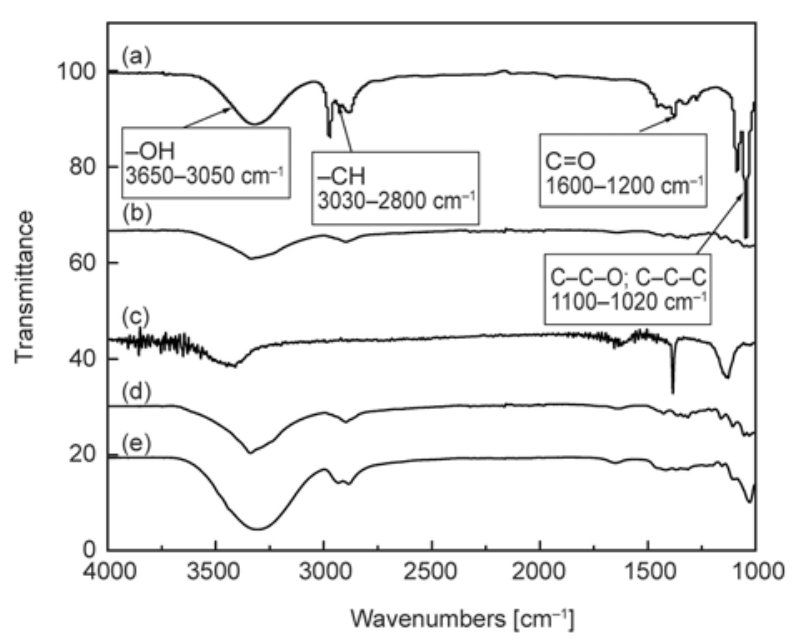

Figure 2. FTIR spectra of (a) GNPs, (b) BC membrane, (c) MWCNTs, (d) GNP-incorporated BC membrane and (e) MWCNT-incorporated BC membrane

in vacuum to eliminate the agglomeration caused by hygroscopic absorption, as well as removing planar water, which would hinder the interaction between MWCNTs and surfactant.

Amino silane coupling agent, $\gamma$-aminopropyl-triethoxysilane (A1100), was obtained from Shanghai Chemical Reagent Co. Ltd., Shanghai, China. NaDDBS and other agents utilized were analytically pure grade and supplied by Sinopharm Chemical Reagent Co. Ltd., Shanghai, China.

\subsection{Preparation of GNPs and GNP membranes}

GNPs were prepared according to the method reported in [14]. As-received GIC particles were subjected to a thermal shock on rapid exposure to $1000^{\circ} \mathrm{C}$ for $20 \mathrm{~s}$ in a muffle furnace, causing GIC particles expanded up to $\sim 40$ times their initial volume with the separation of graphene sheets along the $c$ axis of graphene layers since trapped intercalants within GIC layers decomposed or vapored instantly, and thereby expanded graphite (EG) formed.

EG particles were then dispersed in absolute ethanol and broken down by a $30 \mathrm{~min}$ high speed shear mixing $(2400 \mathrm{r} / \mathrm{min})$ followed by a $12 \mathrm{~h}$ ultrasonic irradiation $(100 \mathrm{~W}, 80 \mathrm{kHz})$ at room temperature, consequently EG particles were effectively fragmented into GNPs. The GNP dispersion was filtered and the obtained GNPs were dried at $110^{\circ} \mathrm{C}$ to remove residue solvents, finally as-prepared GNPs were kept in a dry desiccator for testing and further use. 
As-prepared GNPs $(\sim 6 \mathrm{mg})$ were compressed into disk-like membranes $\left(\varnothing 6 \times 0.2 \mathrm{~mm}^{3}\right)$ for measuring the electrical conductivity of as-prepared GNPs.

\subsection{Preparation of BC-based composite membranes}

Preparation of BC-based composite membranes involved (a) making GNP- or MWCNT-absolute ethanol solutions at $0.05 \mathrm{wt} \%$ concentration, (b) adding no surfactant or surfactant (NaDDBS or A1100, at $0.3 \mathrm{wt} \%$ concentration) to the solutions, (c) dispersing nanofillers (GNPs or MWCNTs) uniformly in the solutions by ultrasonic irradiation $(100 \mathrm{~W}, 80 \mathrm{kHz})$ for $2 \mathrm{~h}$ at room temperature, (d) immersing absolute ethanol-swollen $\mathrm{BC}$ membranes in the resulting even GNP- or MWCNTabsolute ethanol suspensions (note that one membrane in one suspension), (e) keeping the suspensions still for 6,12 , or $24 \mathrm{~h}$, or sonicating the suspensions for $3,6,9,12$ or $15 \mathrm{~h}$ at room temperature, (f) withdrawing, rinsing each membrane thoroughly in absolute ethanol to remove the residual surfactants and unadsorbed GNPs or MWCNTs, and then (g) air-drying at room temperature.

\subsection{Characterization}

Morphological study was carried out using scanning electron microscopy (SEM, JSM-6380LV, JEOL Co., Japan) and two types of field emission SEMs (FESEMs), i.e. LEO1550 (LEO Electron Microscopy Ltd., UK) and Quanta 3D FEG (FEI Co., USA), after the samples were pre-coated with a thin platinum layer. The aspect ratio of GNPs was calculated based on the measured dimensions of 100 GNPs from several FESEM images.

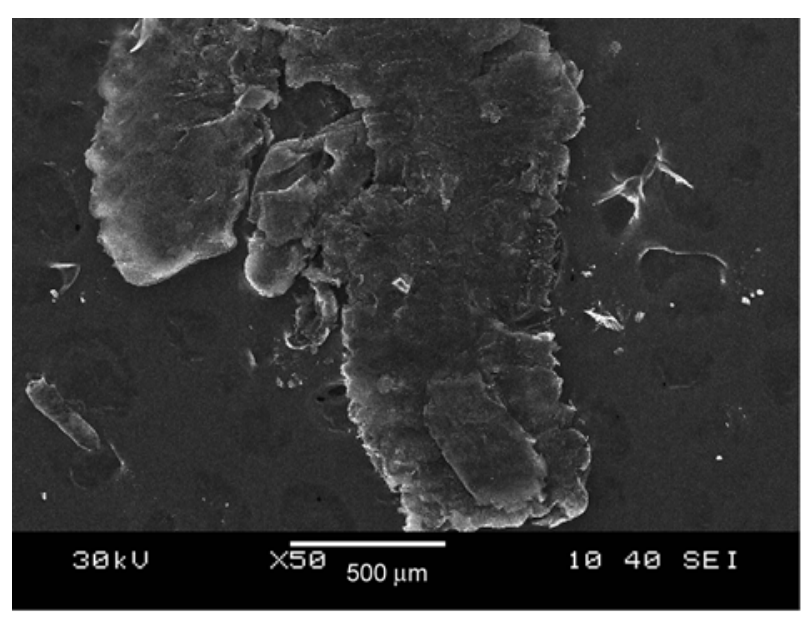

a)
FTIR spectrum was recorded by two types of spectrophotometer, i.e. Thermo Nicolet IS-10 Smart ITR spectrophotometer (Thermo Fisher Scientific Co., Ltd, USA) and Perkin-Elmer Frontier (Perkin Elmer Co., Japan).

The electrical conductivity of as-prepared GNP membranes and BC-based composite membranes was measured using a four-probe with a picoammeter containing an internal voltage source (2611A system, Keithley, USA.) and an impedance measuring meter (HP4284A, HP, USA.). The thickness of membranes was precisely measured for calculating the electrical conductivity. For each measurement, three samples were tested three times at different positions on the BC composite membrane or GNP membrane surface, and then the average was reported.

The amount of GNPs or MWCNTs that were introduced into each $\mathrm{BC}$ membrane was determined by the difference between original mass and residual mass of GNPs or MWCNTs in each absolute ethanol solution.

\section{Results and discussion}

EG particle, as shown in Figure 3a, exhibits a worm-like shape with multiple platelet layers held together at the edge (seen in Figure 3b).

As shown in Figure 4, EG worms were completely torn into GNPs of 3-30 $\mu \mathrm{m}$ in diameter and 20$80 \mathrm{~nm}$ in thickness. GNPs are flat and smooth flakes with sharp corners. Image analysis calculation based on 100 GNPs resulted in an average diameter of $\sim 21 \mu \mathrm{m}$ and thickness of $\sim 47 \mathrm{~nm}$, thereby an aspect ratio of $\sim 447$.

Figure 2a presents the FTIR spectrum of as-prepared GNPs. Characteristic vibrational bands of the

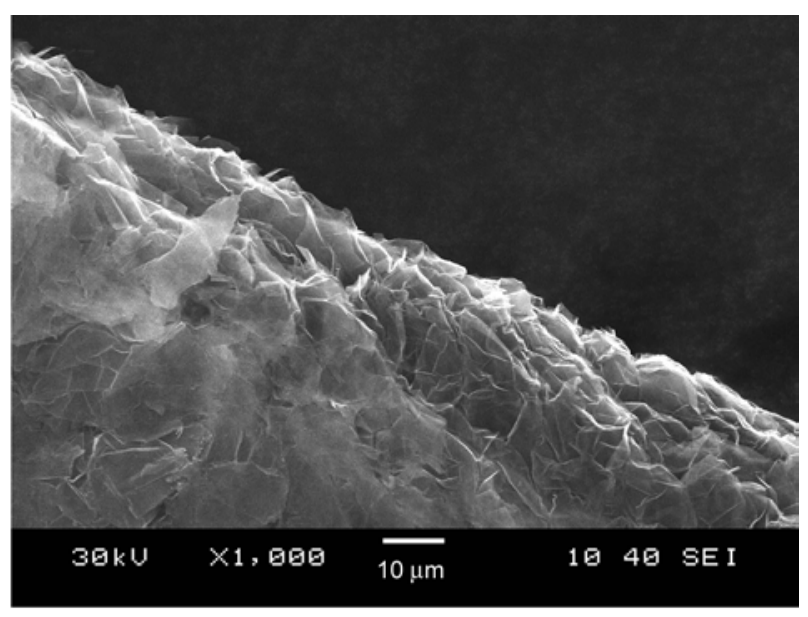

b)

Figure 3. SEM micrographs of an EG particle (a) $50 \times$, (b) $1000 \times$ 


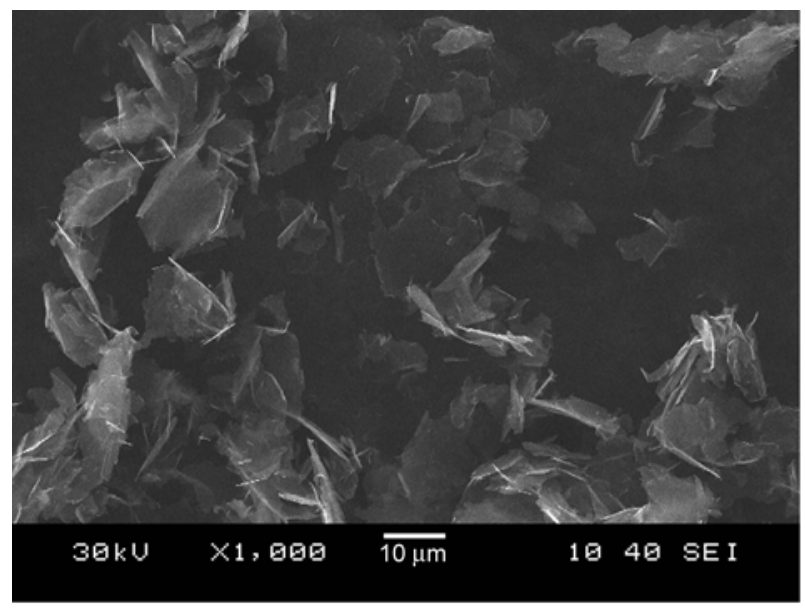

a)

Figure 4. SEM micrographs of GNPs (a) $1000 \times$, (b) $10000 \times$

functional groups are shown in the spectrum. The most characteristic feature is the broad, intense band at $3650-3050 \mathrm{~cm}^{-1}$, suggesting that many hydroxyl groups $(-\mathrm{OH})$ exist on the surface of as-prepared GNPs. Furthermore, the presence of carboxyl functional groups $(\mathrm{C}=\mathrm{O})$ can also be detected. These evidences indicate that during the fabrication of GNPs, treatment of natural graphite with sulfuric acid and thermal shock of GIC particles resulted in some carbon double bonds oxidized, leading to the presence of oxygen-containing functional groups on the GNP flakes, and thereby facilitating the GNP flakes' chemical interactions with surfactant or BC membrane. On the other hand, it is known that oxygen-containing functionalities destroy the aromatic graphene networks and the carbon atom is transformed from a planar $\mathrm{sp}^{2}$-hybridized geometry to a distorted $\mathrm{sp}^{3}$-hybridized geometry, which will be deleterious to the electrical properties of graphite. However, in this work the as-prepared GNPs still exhibited an excellent electrical conductivity, $3.3 \times 10^{2} \mathrm{~S} / \mathrm{cm}$ in basal plane at room temperature, even though it is a value decreased by almost two orders of magnitude in comparison to bulk graphite $\left(\sim 10^{4} \mathrm{~S} / \mathrm{cm}[15]\right)$.

Nanomaterials, including GNPs and MWCNTs, tend to aggregate due to van der Waals attraction and exist in the form of bundles. It is important to separate GNP or MWCNT bundles individually and stabilize them against van der Waals attraction before inserting into $\mathrm{BC}$ membranes for employing the inherent properties of individual nanomaterial as well as facilitating their insertion into the porous BC nanofibril network. A well-dispersed MWCNT suspension was previously achieved by using sur-

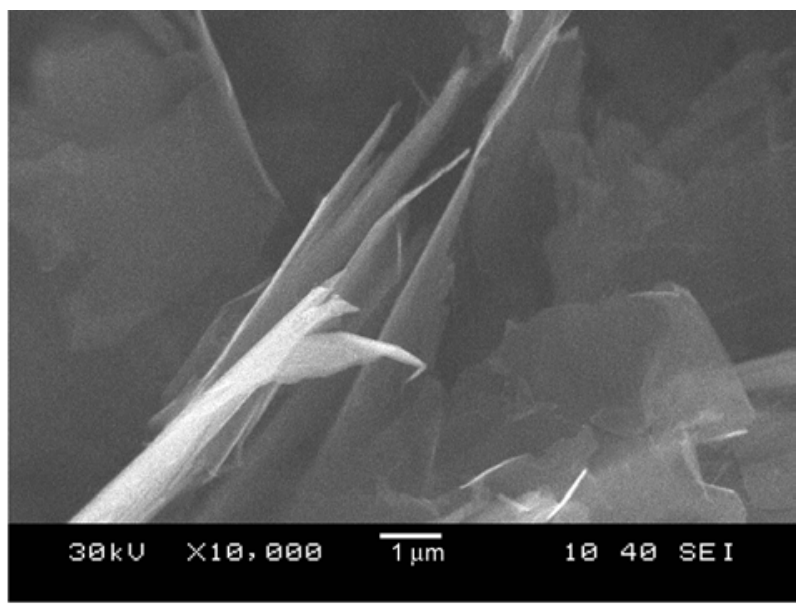

b)

factant NaDDBS [6], and NaDDBS is demonstrated to be more desirable than CTAB as the surfactant for MWCNTs based on the fact that under identical preparation conditions, the electrical conductivity of MWCNT/BC composite membranes obtained by dipping $\mathrm{BC}$ membranes in the NaDDBS-stabilized MWCNT suspension is higher than that of MWCNT/ $\mathrm{BC}$ composite membranes obtained from CTABstabilized MWCNT suspension [5, 6]. Therefore, in this work NaDDBS was used as a reference surfactant. Furthermore, the fabrication process of $\mathrm{BC}$ based composite membranes, both GNP/BC composite membranes and MWCNT/BC composite membranes, was fundamentally similar to the process used in the previous study $[5,6]$ except for the used surfactant and solvent, specifically, in this study A1100 was also chosen as a reference surfactant for comparison and the solvent used is volatile absolute ethanol instead of water.

Generally, nanomaterials are sonicated in the solution of surfactant to facilitate their dispersion. As the concentration of surfactant is too low or too high, large and dense nanomaterial clusters form in the solution even after sonication, but at the optimum surfactant concentration, homogeneous dispersion forms a single phase. A favorable concentration of NaDDBS was reported to be $\sim 0.3 \mathrm{wt} \%$ in the case of $0.05 \mathrm{wt} \%$ MWCNT dispersion [6]. Thus, in this work, $0.3 \mathrm{wt} \%$ of surfactant (NaDDBS or A1100) was studied for the $0.05 \mathrm{wt} \%$ GNP or MWCNT solution for comparison.

A comparison of photographs in Figure 5 indicates that in the case of $0.05 \mathrm{wt} \%$ GNP-absolute ethanol solution, the solution with $0.3 \mathrm{wt} \%$ of surfactant NaDDBS, seen in Figure 5b, has the poorest dis- 


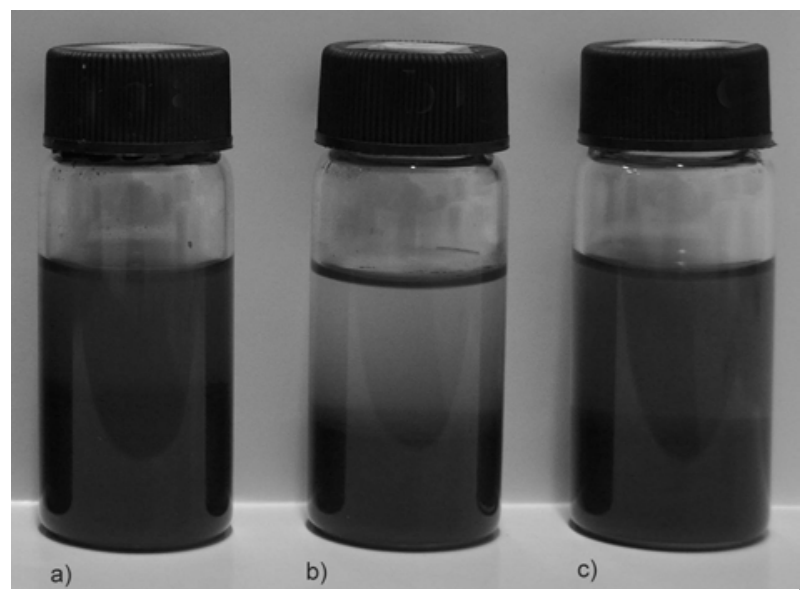

Figure 5. Photographs of $0.05 \mathrm{wt} \%$ GNP-absolute ethanol solution with (a) no surfactant, (b) $0.3 \mathrm{wt} \%$ of surfactant NaDDBS, and (c) $0.3 \mathrm{wt} \%$ of surfactant A1100; 24 h after sonication

persing stability. Interestingly, the solution without surfactant, as is evident in Figure 5a, has the best dispersing stability, performing relatively a little better than that of solution with $0.3 \mathrm{wt} \%$ of surfactant A1100 (Figure 5c). Several literatures have stated that homogeneous colloidal graphene suspensions in aqueous and various organic solvents such as ethanol can be created by simple sonication [16-18], and in this work GNP suspension in absolute ethanol solvent with 24 hour stability was indeed achieved only by sonication without the assistance of surfactants, which avoids the negative effects of surfactants since chemical modification are known to be detrimental to the inherent properties, such as electrical conductivity, of conductive fillers [6], and also makes this technique more easy, economical, and thereby particularly appealing for the industrial practices.

At present, the mechanisms that allow the stable dispersion of graphenes in the organic solvents, such as ethanol, are not clear. Notably, a necessary, but not sufficient, condition seems to be that the solvent molecules be considerably polar [18]. As mentioned above, the as-prepared GNPs in this work were heavily decorated with polar oxygencontaining functionalities, i.e. hydroxyl and carboxyl, which is therefore believed to promote the interaction between GNPs and polar organic solvent ethanol and thereby a good dispersion state of GNPs in ethanol solution. Furthermore, it's a similar case for the surfactant A1100 (molecular formula: $\left.\mathrm{H}_{2} \mathrm{~N}\left(\mathrm{CH}_{2}\right)_{3} \mathrm{Si}\left(\mathrm{OC}_{2} \mathrm{H}_{5}\right)_{3}\right)$ which also has polar groups. The agent, ethanol or A1100, adsorbs onto the surface of GNPs through the so-called 'anchor- ing groups', i.e. hydroxyl groups of ethanol or amine groups of A1100, by van der Waals force or hydrogen bond, while the remainder of the agent (agent chain) extends to the medium to supply steric repulsion for the stabilization of separated GNPs, and it is obvious that when it comes to $0.05 \mathrm{wt} \%$ GNPabsolute ethanol solution, the repulsion offered by ethanol is optimum, but the additional adsorbed surfactant A1100 molecules on the GNP flakes' surface will, on the contrary, add the precipitation burden of GNP flakes and thereby be detrimental to the stabilization of separated GNP flakes. The effect of surfactant NaDDBS on the GNP flakes' dispersing stability will be discussed below.

The as-received MWCNTs utilized in this work were also decorated with polar oxygen-containing functionality, i.e. hydroxyl, which was supposed to also promote a good MWCNT-ethanol interaction and thereby a good dispersion state of MWCNTs in ethanol solution. However, as shown in Figure 6, in the case of $0.05 \mathrm{wt} \%$ MWCNT solution, the steric repulsion provided by organic solvent ethanol only is not strong enough to prevent the reflocculation due to the stronger intertube van der Waals force of MWCNTs than that of GNPs, and solution without the assistance of surfactant, as shown in Figure 6a, yielded larger amount of precipitate compared with the solution with $0.3 \mathrm{wt} \%$ of surfactant A1100. More significantly, surfactant A1100 performed quite well and acted more effectively than surfactant NaDDBS, resulting in a homogeneous black solution with neither sedimentation nor aggregation of MWCNT bundles observed. Consequently, as far as $0.05 \mathrm{wt} \%$ MWCNT-absolute ethanol solution is

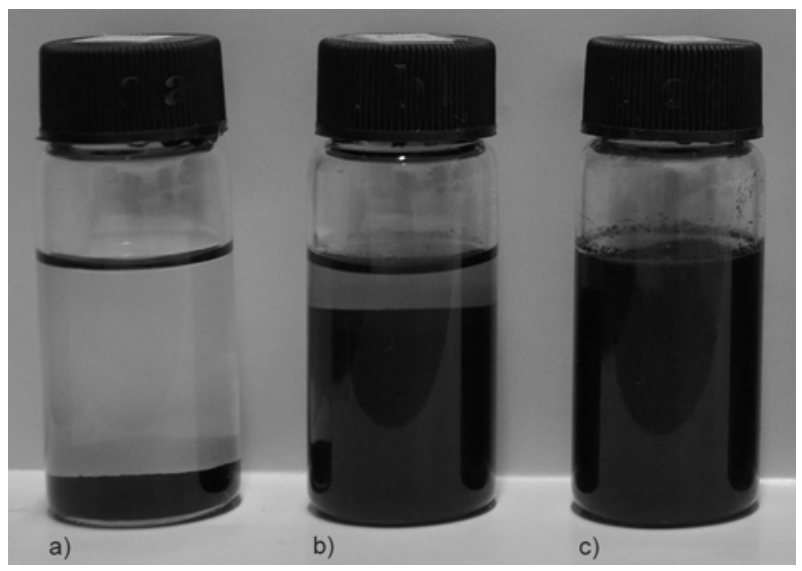

Figure 6. Photographs of $0.05 \mathrm{wt} \%$ MWCNT-absolute ethanol solution with (a) no surfactant, (b) $0.3 \mathrm{wt} \%$ of surfactant NaDDBS, and (c) $0.3 \mathrm{wt} \%$ of surfactant A1100; $24 \mathrm{~h}$ after sonication 
concerned, its stability follows the sequence: solution without surfactant $<$ solution with $0.3 \mathrm{wt} \%$ of surfactant NaDDBS $<$ solution with $0.3 \mathrm{wt} \%$ of surfactant A1100.

NaDDBS (molecular formula: $\mathrm{C}_{12} \mathrm{H}_{25} \mathrm{C}_{6} \mathrm{H}_{4} \mathrm{SO}_{3} \mathrm{Na}$ ), consisting of a benzene ring moiety, an alkyl chain, and a charged group, is a commonly used dispersant for CNTs. Chemical formula of NaDDBS and a suggested schematic of its interaction with a SWCNT can be seen in [19]. It is believed that surfactant NaDDBS form hemimicelles on the CNT surface, and the dispersing capability of NaDDBS to CNT solution can be explained in terms of CNT-NaDDBS interactions, alkyl chain length and headgroup $\left(\mathrm{SO}_{3}^{-}\right)$size. The benzene rings of NaDDBS molecules provide strong CNT-NaDDBS interactions and the $\pi$-like stacking of benzene rings onto the surface of CNTs is believed to increase the binding and surface coverage of surfactant molecules to CNTs significantly. When adsorbing onto the CNT surface, it is probably energetically favorable for the alkyl chains to lie flat along the length of tubes rather than to bend around the circumference. CNT stability depends particularly on those surfactant molecules that lie along the tube surface, parallel to the tube central axis, since the electrostatic repulsion of $\mathrm{SO}_{3}^{-}$leads to the charge stabilization of tubes by screened Coulomb interactions [19]. Therefore, we suppose that in this work the different effects, i.e. NaDDBS exhibiting positive effect on the stabilization of MWCNTs whereas little effect on the stabilization of GNPs, arise from different

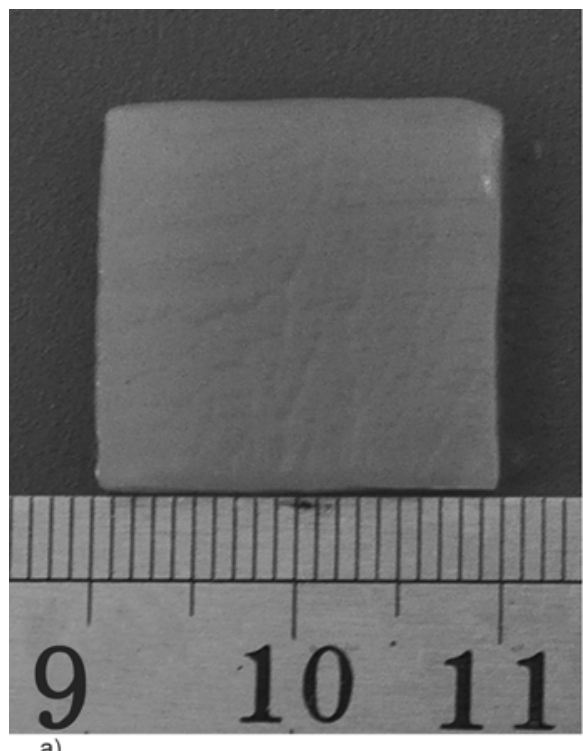

a) packing density. The larger dimensional size of 2-D GNPs offer more difficulty to achieve high surface packing density of surfactant NaDDBS, resulting in screened Coulomb interactions not enough to stabilize GNPs against van der Waals attraction. However, previous knowledge of the dispersion behavior of CNTs or GNPs in organic solvents or surfactants can not provide much more insight into this question because the solvent or surfactant characteristics required to disperse CNTs or GNPs efficiently have not yet been well established. Clarifying the dispersion behavior of GNPs will necessitate further in-depth investigations.

Thus far, as the result of evaluating the competing stabilization characteristics, a stable $0.05 \mathrm{wt} \%$ GNPabsolute ethanol suspension (with no surfactant), as well as a stable $0.05 \mathrm{wt} \%$ MWCNT-absolute ethanol suspension with the assistance of $0.3 \mathrm{wt} \%$ of surfactant A1100, was chosen as the base suspension for the following study. Then gelatinous absolute ethanol-swollen pristine $\mathrm{BC}$ membranes were immersed or sonicated in the base suspensions (note that one membrane in one suspension), consequently, the white pristine membranes (Figure 7a) turned into black composite membranes(Figure 7b) and there are no obvious appearance difference between the obtained GNP/BC composite membranes and MWCNT/BC composite membranes.

Figure 8a presents the surface morphology of GNPincorporated $\mathrm{BC}$ membrane with the surface morphology of MWCNT-incorporated BC membrane also shown in Figure 8b. After air-dried at room

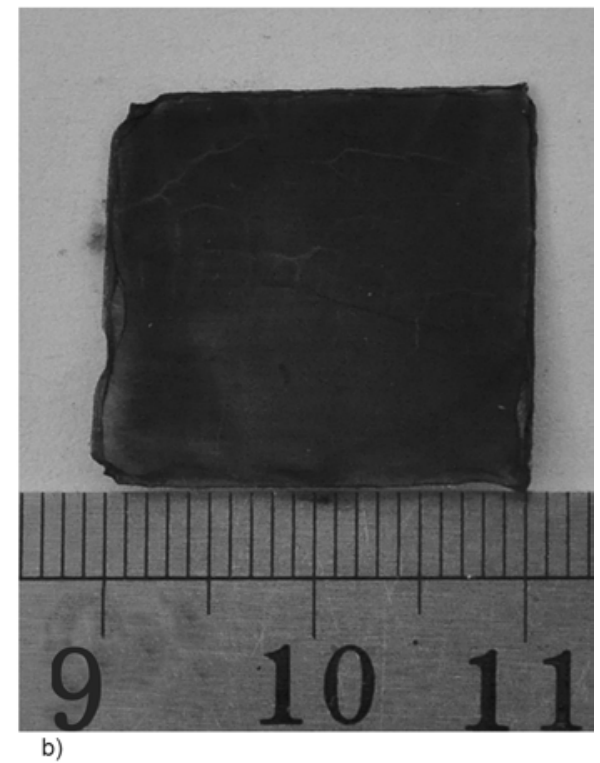

Figure 7. Photographs of (a) absolute ethanol-swollen BC membrane and (b) air-dried GNP/BC composite membrane; sonication for $9 \mathrm{~h}$ 


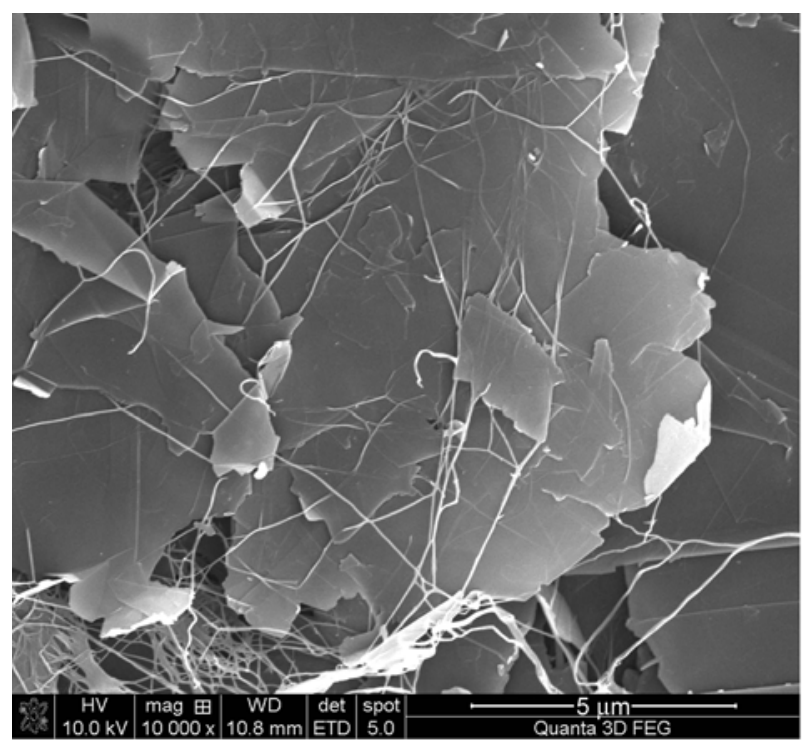

a)

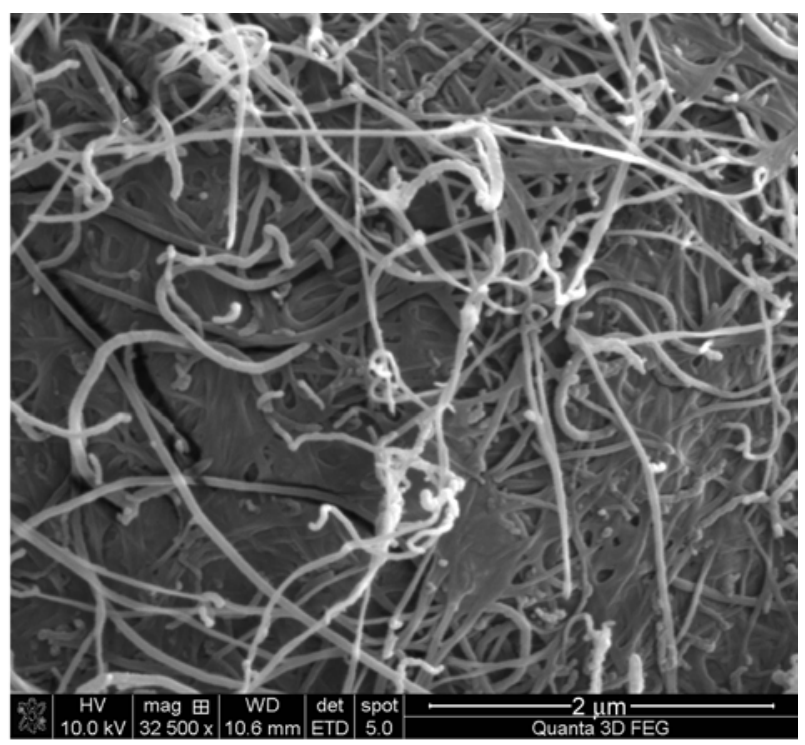

b)

Figure 8. FESEM micrographs of the surface of (a) GNP-incorporated BC membrane and (b) MWCNT-incorporated BC membrane; sonication for $9 \mathrm{~h}$

temperature, the porous structure of BC membranes collapsed into a dense structure with incorporated GNP or MWCNT particles relatively loose-structured over the membrane surface. The diameter of GNPs further decreased to be less than $15 \mu \mathrm{m}$ after the additional $9 \mathrm{~h}$ sonication treatment during the preparation of BC-based composite membranes.

Both flake-shaped GNPs and rod-shaped MWCNTs were seen effectively inserting in the surface layer of BC membranes. Flake-shaped GNPs, weaving through the surface layer of weblike cellulose nanofibrils, were indeed fixed or trapped by adjacent nanofibrils in the BC surface network. Meanwhile, rod-shaped MWCNTs were inserted into BC membranes with each remainder part of MWCNTs spreading on the membrane surface, or entangling with other MWCNTs or cellulose nanofibrils. Furthermore, GNPs exhibited larger surface coverage in the surface layer of $\mathrm{BC}$ membrane due to their nearly fully extended 2-D morphology and thereby were supposed to provide more electrical conductivity reinforcement than 1-D MWCNTs.

The cross section of GNP- or MWCNT-incorporated $\mathrm{BC}$ membranes were also examined by FESEM after fractured in liquid nitrogen. Figure 9 shows that ultrafine $\mathrm{BC}$ ribbons form a dense reticulated 'layer by layer' structure, and the thickness of GNPs further decreased to be less than $50 \mathrm{~nm}$ after the additional $9 \mathrm{~h}$ sonication treatment during the preparation of $\mathrm{BC}$-based composite membranes. Two different domains could be distinguished in
Figure 9, a continuous network of cellulose nanofibrils and several inserted GNPs or MWCNTs shown by arrows. More importantly, Figure 9 also gives a direct proof that $\mathrm{BC}$ membrane acted as a template or a supporting framework, flake-shaped GNPs weaved through the surface layer of $\mathrm{BC}$ membrane and covered over it, while rod-shaped MWCNTs homogeneously inserted into the $\mathrm{BC}$ membrane, maintaining their dispersity without aggregation, through the pore structures and tunnels within the cellulose membrane.

Figure 10 presents the time dependence of the electrical conductivity of $\mathrm{BC}$ membranes incorporated with GNP or MWCNT particles. The electrical conductivity of these $\mathrm{BC}$ composite membranes increases with the increasing immersion or sonication time, indicating that more GNP or MWCNT particles would be incorporated in the membranes after longer immersion or sonication time. Before the capacity limitation of $\mathrm{BC}$ membrane is reached, longer immersion time, generally, means more opportunity for the particles to be incorporated. Moreover, the micro-currents of sonication fluid, shear forces and oscillating cavitation bubbles induced by the high acoustic intensity of ultrasound bath may remarkably facilitate GNP flake's or MWCNT rod's insertion and entanglement into $\mathrm{BC}$ membranes, thus more particles would be incorporated with longer sonication time.

It is well known that $\mathrm{BC}$ is stabilized by extensive hydrogen bonding, and there are a mass of hydroxyl 


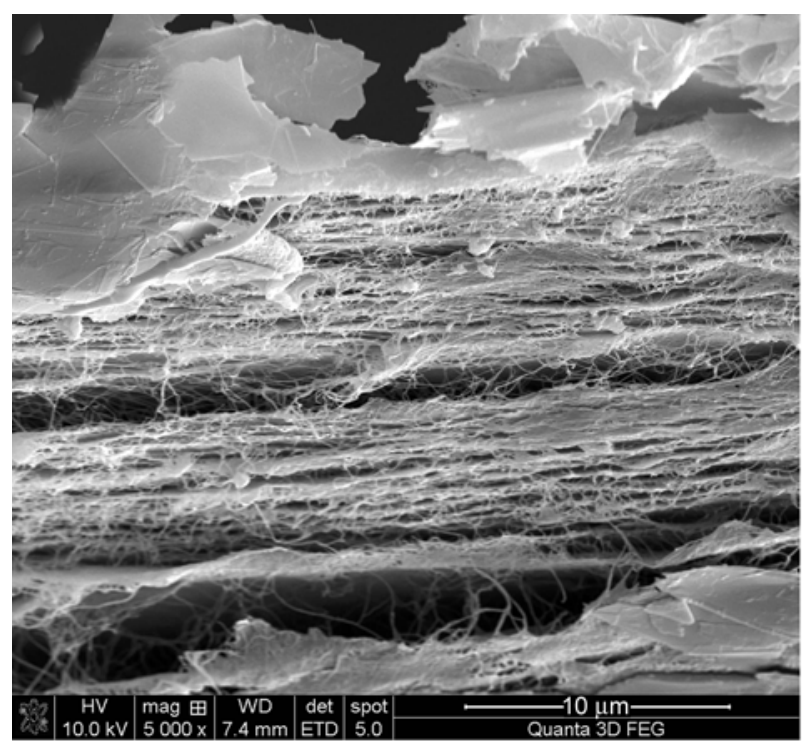

a)

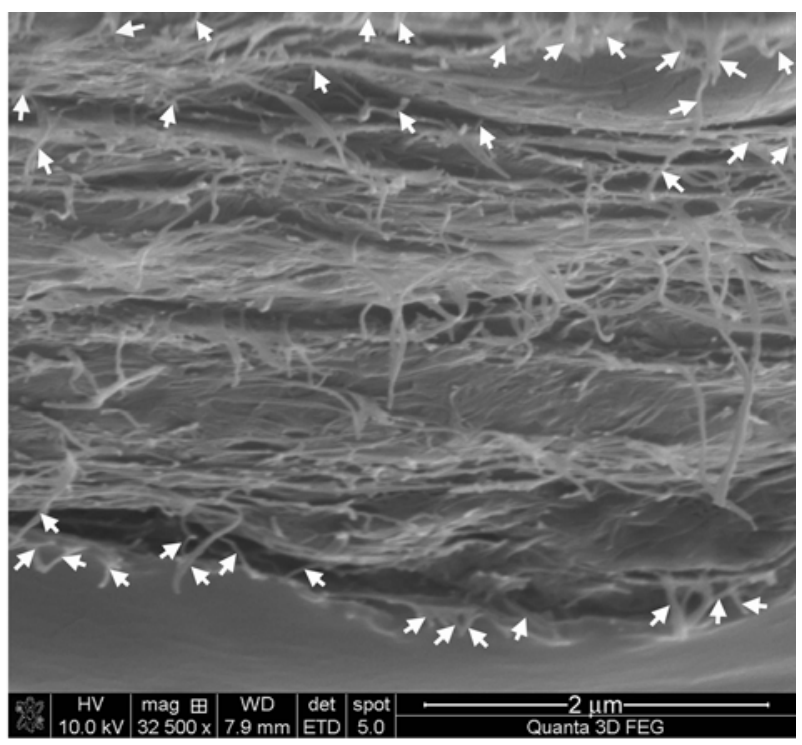

b)

Figure 9. FESEM micrographs of the cross section of (a) GNP-incorporated BC membrane and (b) MWCNT-incorporated $\mathrm{BC}$ membrane, arrows show MWCNTs; sonication for $9 \mathrm{~h}$

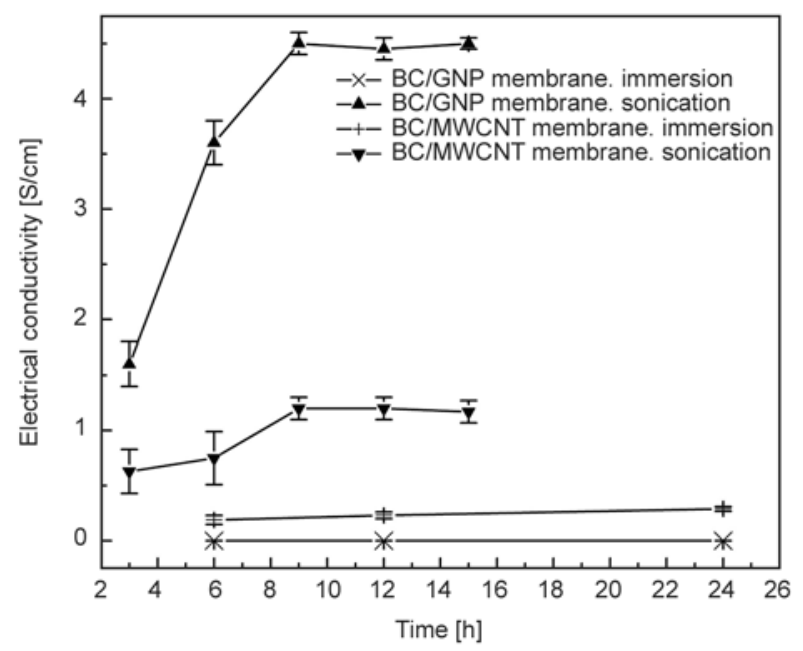

Figure 10. Electrical conductivity of GNP/BC or MWCNT/ $\mathrm{BC}$ composite membranes at room temperature. Lines are given only for showing the tendency.

groups in the $\mathrm{BC}$ membrane (Figure $2 \mathrm{~b}$ ). In this work, as shown in Figure 2, GNP or MWCNT particles, on the surface of which many hydroxyl groups were detected by FTIR, are therefore supposed to be covalently attached by etherification with the cellulose nanofibrils. But it is hard to confirm this strong interaction, i.e. ether linkage, exists between the cellulose nanofibrils and the particles of GNP or MWCNT by FTIR since massive ether linkages already exist among the cellulose nanofibrils. However, in this work, sonication-prepared BC composite membranes were found exhibiting more considerable increase of electrical conductivity than immersion-prepared ones, which not only proved that sonication method is more useful than immersion method in remarkably facilitating GNP flake's or MWCNT rod's insertion and entanglement into $\mathrm{BC}$ membranes but also provided a direct evidence that strong physical and chemical interaction, i.e. chemical ether linkage and physical tangling between the BC nanofibrils and the particles of GNP or MWCNT, exists even after $15 \mathrm{~h}$ sonication.

As shown in Figure 10, the electrical conductivity of sonication-prepared GNP/BC composite membranes increased from 1.6 to $4.5 \mathrm{~S} / \mathrm{cm}$ while that of sonication-prepared MWCNT/BC composite membranes increased from $6.3 \times 10^{-1}$ to $1.2 \mathrm{~S} / \mathrm{cm}$, then there is no obvious increase of electrical conductivity of $\mathrm{BC}$ composite membranes as the sonication time sequentially increased from 9 to $15 \mathrm{~h}$, which can be attributed to the capacity limitation of $\mathrm{BC}$ membranes for the incorporated GNP or MWCNT particles. The deviation of data in Figure 10 is within an acceptable degree considering the small error bar for the maximum data points, demonstrating that GNP or MWCNT particles were really incorporated uniformly and densely in the $\mathrm{BC}$ membranes since it is known that homogeneous dispersion and high amount of conductive particles in matrix are the two most important requirements for composites to achieve a uniform and high electrical conductivity. It is well known that the electrical conductivity of $\mathrm{BC}$ composite membranes depends on the amount of electrical pathways formed by conducting GNP or MWCNT particles incorporated in the BC nanofib- 
ril network. GNPs have advantage in forming conducting pathways due to their 2-D morphology and thus supposedly allow the electrical conductivity of composite membranes to be enhanced to a greater extent than other spherical or rod-like conducting particles. An attempt was made to determine the amount of GNP or MWCNT particles incorporated in the $\mathrm{BC}$ membranes using Thermogravimetric Analysis under a nitrogen atmosphere, but this measurement was unsuccessful and the cause was proved to be in accord with the explanation previously reported [5], i.e. the $\mathrm{BC}$ membrane charred after burning up to about $600^{\circ} \mathrm{C}$ and did not decompose completely. However, in this work the amount of GNP or MWCNT particles that were introduced into each BC membrane was successfully decided by the difference between original mass and residual mass of GNP or MWCNT particles in each absolute ethanol solution. GNP/BC composite membrane with the maximum electrical conductivity $4.5 \mathrm{~S} / \mathrm{cm}$ was found to contain $8.7 \mathrm{wt} \% \mathrm{GNPs}$, while MWCNT/ BC composite membrane with the maximum electrical conductivity $1.2 \mathrm{~S} / \mathrm{cm}$, which surpasses the maximum electrical conductivity $1.4 \times 10^{-1} \mathrm{~S} / \mathrm{cm}$ of immersion-prepared $\mathrm{BC}$ composite membrane incorporated with $9.6 \mathrm{wt} \%$ MWCNTs reported by elemental analysis in Ref. [5], was found to be incorporated by $13.9 \mathrm{wt} \%$ MWCNTs. Therefore, compared with 1-D MWCNTs, as long as GNPs inserted into BC membranes, the 2-D reinforcement of GNPs was proven to be more effective in improving the electrical conductivity of $\mathrm{BC}$ membranes thus not only break the bottleneck of further improving the electrical conductivity of BC-based composite membranes but also broaden the applications of $\mathrm{BC}$ and GNPs.

\section{Conclusions}

In this study, BC-based composite membranes were prepared by immersing or sonicating gelatinous absolute ethanol-swollen pristine $\mathrm{BC}$ membranes in stable $0.05 \mathrm{wt} \%$ GNP-absolute ethanol suspensions or $0.05 \mathrm{wt} \%$ MWCNT-absolute ethanol suspensions stabilized with the assistance of $0.3 \mathrm{wt} \%$ of A1100. By physical and chemical methods, flake-shaped GNPs, weaving through the surface layer of weblike cellulose nanofibrils, were indeed fixed or trapped by the adjacent nanofibrils in the $\mathrm{BC}$ surface network, while rod-shaped MWCNTs were homogeneously inserted into the $\mathrm{BC}$ membrane through pore structures and tunnels within the $\mathrm{BC}$ membrane.

Sonication-prepared BC composite membranes exhibit more considerable increase of electrical conductivity than immersion-prepared ones, which not only proved that sonication is a more useful method than immersion to remarkably facilitate GNP flake's or MWCNT rod's insertion into BC membranes but also provided a direct evidence that strong interaction, i.e. chemical ether linkage and physical tangling between the $\mathrm{BC}$ nanofibrils and the particles of GNP or MWCNT, exists even after $15 \mathrm{~h}$ sonication in absolute ethanol.

Furthermore, compared with 1-D MWCNTs, as long as GNPs inserted into BC membranes, the 2-D reinforcement of GNPs was proved more effectively in improving the electrical conductivity of pristine $\mathrm{BC}$ membranes. $\mathrm{BC}$ membrane with $8.7 \mathrm{wt} \%$ incorporated GNPs reached the maximum electrical conductivity of $4.5 \mathrm{~S} / \mathrm{cm}$, while MWCNT/BC composite membrane containing $13.9 \mathrm{wt} \%$ MWCNTs achieved the maximum electrical conductivity of $1.2 \mathrm{~S} / \mathrm{cm}$, thus not only break the bottleneck of further improving the electrical conductivity of BCbased composite membranes but also broaden the applications of BC and GNPs.

\section{Acknowledgements}

The authors are grateful for the financial support of National Natural Science Foundation of China (No. 51002077, No. 51203074), Fundamental Research Funds for the Central Universities (No. NUST 2011YBXM163), Jiangsu Overseas Research \& Training Program for University Prominent Young \& Middle-aged Teachers and Presidents, and Special Foundation for 'first-grade Zijin's Star' of 'Excellence initiative' Project of Nanjing University of Science and Technology (No.AB41339).

\section{References}

[1] Chen P., Cho S. Y., Jin H-J.: Modification and applications of bacterial celluloses in polymer science. Macromolecular Research, 18, 309-320 (2010). DOI: $10.1007 / \mathrm{s} 13233-010-0404-5$

[2] Siró I., Plackett D.: Microfibrillated cellulose and new nanocomposite materials: A review. Cellulose, 17, 459494 (2010). DOI: $10.1007 / \mathrm{s} 10570-010-9405-\mathrm{y}$

[3] Ifuku S., Nogi M., Abe K., Handa K., Nakatsubo F., Yano H.: Surface modification of bacterial cellulose nanofibers for property enhancement of optically transparent composites: Dependence on acetyl-group DS. Biomacromolecules, 8, 1973-1978 (2007).

DOI: $\underline{10.1021 / \mathrm{bm} 070113 \mathrm{~b}}$ 
[4] Yano H., Sugiyama J., Nakagaito A. N., Nogi M., Matsuura T., Hikita M., Handa K.: Optically transparent composites reinforced with networks of bacterial nanofibers. Advanced Materials, 17, 153-155 (2005).

DOI: $10.1002 /$ adma.200400597

[5] Yoon S. H., Jin H-J., Kook M-C., Pyun Y. R.: Electrically conductive bacterial cellulose by incorporation of carbon nanotubes. Biomacromolecules, 7, 1280-1284 (2006).

DOI: $10.1021 / \mathrm{bm} 050597 \mathrm{~g}$

[6] Yoon S. H., Kang M., Park W-I., Jin H-J.: Electrically conductive polymeric membranes by incorporation of carbon nanotubes. Molecular Crystals and Liquid Crystals, 464, 685-690 (2007).

DOI: $10.1080 / 15421400601030449$

[7] Jung R., Kim H-S., Kim Y., Kwon S-M., Lee H. S., Jin H-J.: Electrically conductive transparent papers using multiwalled carbon nanotubes. Journal of Polymer Science Part B: Polymer Physics, 46, 1235-1242 (2008). DOI: $10.1002 /$ polb.21457

[8] Kim Y., Kim H-S., Bak H., Yun Y. S., Cho S. Y., Jin HJ.: Transparent conducting films based on nanofibrous polymeric membranes and single-walled carbon nanotubes. Journal of Applied Polymer Science, 114, 2864 2872 (2009).

DOI: $10.1002 / a p p .30829$

[9] Kim Y., Kim H-S., Yun Y. S., Bak H., Jin H-J.: Agdoped multiwalled carbon nanotube/polymer composite electrodes. Journal of Nanoscience and Nanotechnology, 10, 3571-3575 (2010).

DOI: $10.1166 /$ jnn.2010.2232

[10] Yun Y. S., Bak H., Jin H-J.: Porous carbon nanotube electrodes supported by natural polymeric membranes for PEMFC. Synthetic Metals, 160, 561-565 (2010). DOI: 10.1016/j.synthmet.2009.12.003

[11] Feng Y., Zhang X., Shen Y., Yoshino K., Feng W.: A mechanically strong, flexible and conductive film based on bacterial cellulose/graphene nanocomposite. Carbohydrate Polymers, 87, 644-649 (2011).

DOI: $10.1016 /$ j.carbpol.2011.08.039
[12] Yan J., Wei T., Shao B., Ma F., Fan Z., Zhang M., Zheng C., Shang Y., Qian W., Wei F.: Electrochemical properties of graphene nanosheet/carbon black composites as electrodes for supercapacitors. Carbon, 48, 17311737 (2010).

DOI: $10.1016 /$ j.carbon.2010.01.014

[13] Zhou T., Wang X., Liu X., Xiong D.: Influence of multi-walled carbon nanotubes on the cure behavior of epoxy-imidazole system. Carbon, 47, 1112-1118 (2009). DOI: $10.1016 /$ j.carbon.2008.12.039

[14] Chen G., Weng W., Wu D., Wu C., Lu J., Wang P., Chen X.: Preparation and characterization of graphite nanosheets from ultrasonic powdering technique. Carbon, 42, 753-759 (2004).

DOI: $10.1016 /$ j.carbon.2003.12.074

[15] Li J., Sham M. L., Kim J-K., Marom G.: Morphology and properties of UV/ozone treated graphite nanoplatelet/epoxy nanocomposites. Composites Science and Technology, 67, 296-305 (2007).

DOI: $10.1016 /$ j.compscitech.2006.08.009

[16] Potts J. R., Dreyer D. R., Bielawski C. W., Ruoff R. S.: Graphene-based polymer nanocomposites. Polymer, 52, 5-25 (2011). DOI: $10.1016 /$ j.polymer.2010.11.042

[17] Park S., Ruoff R. S.: Chemical methods for the production of graphenes. Nature Nanotechnology, 4, $217-$ 224 (2009). DOI: 10.1038/nnano.2009.58

[18] Paredes J. I., Villar-Rodil S., Martínez-Alonso A., Tascón J. M. D.: Graphene oxide dispersions in organic solvents. Langmuir, 24, 10560-10564 (2008). DOI: $10.1021 / 1 a 801744 a$

[19] Islam M. F., Rojas E., Bergey D. M., Johnson A. T., Yodh A. G.: High weight fraction surfactant solubilization of single-wall carbon nanotubes in water. Nano Letters, 3, 269-273 (2003). DOI: $\underline{10.1021 / \mathrm{nl} 1025924 \mathrm{u}}$ 